\title{
PRIME FACTOR ALGEBRAS OF THE COORDINATE RING OF QUANTUM MATRICES
}

\author{
K. R. GOODEARL AND E. S. LETZTER
}

(Communicated by Maurice Auslander)

\begin{abstract}
It is proved that every prime factor algebra of the coordinate ring $\mathscr{Q}_{q}\left(M_{n}(k)\right)$ of quantum $n \times n$ matrices over a field $k$ is an integral domain (albeit not necessarily commutative) when $q$ is not a root of unity. The same conclusion follows for the quantum groups $\mathscr{O}_{q}\left(\mathrm{SL}_{n}(k)\right)$ and $\mathscr{O}_{q}\left(\mathrm{GL}_{n}(k)\right)$. The proof uses a $q$-analog of Sigurdsson's theorem bounding the Goldie ranks of prime factors of differential operator rings; this $q$-analog in turn is based on results from the authors' recent work on $q$-skew polynomial rings.
\end{abstract}

\section{INTRODUCTION}

It is well known that the quantum coordinate rings $\mathscr{O}_{q}\left(M_{n}(k)\right)$ can be expressed as iterated skew polynomial rings over the field $k$ (see $\S 3$ for details). In this regard, the structure of $\mathscr{O}_{q}\left(M_{n}(k)\right)$ is parallel to that of enveloping algebras of completely solvable finite dimensional Lie algebras over $k$, since such enveloping algebras are iterated differential operator extensions of $k$. Sigurdsson used the latter fact, together with his theorem giving bounds for the Goldie ranks of prime factors of certain differential operator rings [19, 2.1], to give a ring-theoretic proof of Dixmier's theorem that prime factors of enveloping algebras of solvable finite dimensional complex Lie algebras are integral domains $[19,2.7]$. We construct a parallel route to show that prime factors of $\mathscr{O}_{q}\left(M_{n}(k)\right)$ are integral domains provided that $q$ is not a root of unity. In order to control the Goldie ranks of the prime factors of the relevant skew polynomial rings, we establish a $q$-analog of Sigurdsson's theorem. Such a result does not hold for skew polynomial rings $R[y ; \tau, \delta]$ in general (not even in characteristic zero) but does hold under the assumption that $(\tau, \delta)$ is a q-skew derivation for a suitable $(\tau, \delta)$-constant $q$, meaning that $\delta \tau=q \tau \delta$. For this case, we derive the result from our analysis in [6] of prime ideals in $q$-skew polynomial rings.

The first section of the paper is devoted to our $q$-analog of Sigurdsson's theorem. An extension to a class of iterated skew polynomial rings is obtained

Received by the editors May 8, 1992 and, in revised form, November 16, 1992; this paper was presented in the Special Session on Quantum Groups and Regular Algebras at the $876^{\text {th }}$ meeting of the American Mathematical Society.

1991 Mathematics Subject Classification. Primary 16D30, 16P40, 16 S36.

The research of the first author was partially supported by a National Science Foundation research grant and that of the second author by a National Science Foundation postdoctoral research fellowship. 
in $\S 2$, and the applications to the quantum coordinate rings $\mathscr{O}_{q}\left(M_{n}(k)\right)$ follow in $\S 3$.

\section{A $q$-ANalog to a theorem of Sigurdsson}

Assume throughout this section that $R$ is a noetherian ring, that $\tau$ is an automorphism of $R$, and that $\delta$ is a (left) $\tau$-derivation of $R$. (The reader is referred to [7] or [15] for unexplained terminology and notation.) We investigate the Goldie ranks of prime factor rings of the skew polynomial ring (Ore extension) $R[y ; \tau, \delta]$. For the applications to quantum matrices, the rank 1 case is the important one, due to the fact that a prime noetherian ring is an integral domain if and only if its Goldie rank is 1 (see 1.1(iv)).

1.1. We review a few basic facts about (Goldie) rank.

(i) A module $A$ has finite rank $n$ if and only if $A$ has an essential submodule which is a direct sum of $n$ uniform submodules $[7,4.16 ; 15,2.2 .9]$.

(ii) Goldie rank is preserved under localization with respect to an Ore set of regular elements [15, 2.2.12].

(iii) A prime noetherian ring $T$ has a simple artinian ring of fractions, Fract $(T)$, by Goldie's Theorem, and $\operatorname{Fract}(T)$ and $T$ have the same rank (say as right modules), by (ii). Moreover, $\operatorname{Fract}(T) \cong M_{n}(D)$ for some positive integer $n$ and some division ring $D$. Since $M_{n}(D)$ is a direct sum of $n$ simple right ideals, its rank is $n$ by (i), and thus $\operatorname{rank}(T)=n$. In particular, it follows that $T$ has the same rank as a right or a left module.

(iv) It is clear from (iii) that a prime noetherian ring with rank 1 must be an integral domain, since its ring of fractions is a division ring. On the other hand, every noetherian integral domain has rank $1[7,5.15 ; 15,2.2 .11(\mathrm{i})]$.

Lemma 1.2. Let $S=R[y ; \tau, \delta]$. If $R$ is prime, then the Goldie ranks of $R$ and $S$ are equal.

Proof. Since $R$ is prime, the set $\mathscr{C}$ of regular elements of $R$ is an Ore set in $R$ and $R \mathscr{C}^{-1}=\operatorname{Fract}(R)$ is a simple artinian ring. By, e.g., $[4,1.4], \mathscr{C}$ is also an Ore set in $S$, and $S \mathscr{C}^{-1} \cong\left(R \mathscr{C}^{-1}\right)[y ; \tau, \delta]$. Consequently, in view of 1.1(ii), there is no loss of generality in assuming $R$ to be simple artinian. Thus $R_{R}$ is isomorphic to a direct sum of $\operatorname{rank}(R)$ copies of a minimal right ideal $A$ of $R$, so $S_{S}$ is isomorphic to a direct sum of $\operatorname{rank}(R)$ copies of $A S$. Hence, in order to prove the lemma, it suffices to show that $A S$ is a uniform right ideal of $S$.

Now choose a uniform right ideal $U \subseteq A S$ and a nonzero element $u \in U$ of minimal degree as a polynomial in $y$, say degree $d$. Let $a$ be the leading coefficient of $u$. Then $a R=A$, and so $\mathrm{r} \operatorname{ann}_{R}(a)$ is a maximal right ideal of $R$. Consequently, the right ideal $B=\tau^{-d}\left(\mathrm{r} \cdot \operatorname{ann}_{R}(a)\right)$ is also maximal; in particular, $R / B \cong A$. For any $b \in B$ we have $a \tau^{d}(b)=0$; whence, $\operatorname{deg}(u b)<$ $d$, and so $u b=0$. Thus $u B S=0$. It follows with an easy induction on degree that $\mathrm{r}$. $\operatorname{ann}_{S}(u)=B S$, and so $u S \cong S / B S \cong(R / B) \otimes_{R} S \cong A S$. Therefore, $A S$ is uniform, as desired.

The following result generalizes $[19,2.1]$. Recall that a prime ideal $P$ in a ring $T$ is said to be completely prime provided the factor ring $T / P$ is an integral domain. 
Theorem 1.3. Suppose that $\delta \tau=q \tau \delta$ for some central unit $q \in R$ such that $\delta(q)=0$ and $\tau(q)=q$, and suppose that $q^{i}+q^{i-1}+\cdots+1$ is invertible in $R$ for all positive integers $i$. Further assume that there is a finite upper bound $b$ for the Goldie ranks of all prime factors of $R[z ; \tau]$. Then each prime factor of the ring $S=R[y ; \tau, \delta]$ has Goldie rank at most $b$. In particular, if all prime ideals of $R[z ; \tau]$ are completely prime, then all prime ideals of $S$ are completely prime.

Proof. Given $P \in \operatorname{spec} S$, choose $Q \in \operatorname{spec} R$ minimal over $P \cap R$, so that $P$ lies over $Q$. If the $\tau$-orbit of $Q$ is infinite, then $\operatorname{rank}(S / P)=\operatorname{rank}(R / Q)$ by $[6,8.5 . I]$. Since $R / Q$ is a homomorphic image of $R[z ; \tau]$, it is immediate that $\operatorname{rank}(S / P) \leq b$ in this case.

Now assume that the $\tau$-orbit of $Q$ is finite, set $J=\bigcap_{i} \tau^{i}(Q)$, and note that $J$ is $\tau$-prime [3, Remarks $4^{*}, 5^{*}$, p. 338]. By [6, 10.3(i),(ii)], $J$ is $\delta$-stable as well as $\tau$-stable, and $P \supset J S$. Hence, we may assume without loss of generality that $J=0$. Now $R$ is a $\tau$-prime ring, and $Q$ is a minimal prime ideal of $R$. Since the intersection $P \cap R$ is contained in a minimal prime ideal, it is disjoint from the set $\mathscr{C}$ of regular elements of $R$ (e.g., $[7,6.3]$ ). Thus $P$ induces a prime ideal in the localization $S \mathscr{C}^{-1}$. Moreover, $S \mathscr{C}^{-1} \cong A[y ; \tau, \delta]$, where $A=R \mathscr{C}^{-1}[4,1.4]$. In particular, the Goldie rank of $S / P$ equals that of some prime factor of $A[y ; \tau, \delta]$. Since the prime factors of $A[z ; \tau]$ all have Goldie rank at most $b$, we may now assume that $R=A$. Thus, there is no loss of generality in assuming that $R$ is artinian and $\tau$-simple.

If either $R$ is not simple or $P \neq 0$, it follows from [6,3.6, 10.3(iii)] that $\delta$ is an inner $\tau$-derivation. In these cases, $S \cong R[z ; \tau]$ (e.g., $[4,1.5 \mathrm{c}]$ ), and we are done. On the other hand, if $R$ is simple and $P=0$, we conclude from Lemma 1.2 that $\operatorname{rank}(S / P)=\operatorname{rank}(R) \leq b$.

In the remainder of this section we provide a result analogous to Theorem 1.3 for the case when $q$ is a root of unity and $R$ is a $\mathbb{Q}$-algebra.

Lemma 1.4. Let $R \subseteq S$ be an embedding of noetherian rings such that $S$ can be generated as a right $R$-module by $t$ elements. If there is a finite upper bound $b$ for the Goldie ranks of all prime factors of $R$, then the Goldie ranks of the prime factors of $S$ do not exceed $t b$.

Proof. Let $P \in \operatorname{spec} S$, and set $F=\operatorname{Fract}(S / P)$. If we choose $Q \in \operatorname{spec} R$ to be the right annihilator of some simple $F-R$-bimodule factor of $F$, then $\operatorname{rank}(S / P) \leq t \operatorname{rank}(R / Q)$ by $[11,3.7]$. The lemma follows.

Theorem 1.5. Suppose that $R$ is a $\mathbb{Q}$-algebra and that $\delta \tau=q \tau \delta$ for some central unit $q \in R$ such that $\delta(q)=0$ and $\tau(q)=q$. Assume that there exists an integer $t>1$ such that $q^{t-1}+q^{t-2}+\cdots+1=0$ while $q^{i-1}+q^{i-2}+\cdots+1$ is invertible in $R$ for $i=1, \ldots, t-1$. Further assume that there is a finite upper bound $b$ for the Goldie ranks of all prime factors of $R\left[z ; \tau^{t}\right]$. Then all prime factors of the ring $S=R[y ; \tau, \delta]$ have Goldie rank at most $t b$.

Proof. Let $U$ denote the subring of $S$ generated by $R \cup\left\{y^{t}\right\}$. In $[6,3.8]$ it is shown that the pair $\left(\tau^{t}, \delta^{t}\right)$ is a skew derivation of $R$ satisfying the relation $\delta^{t} \tau^{t}=\tau^{t} \delta^{t}$, that the ring $U$ is equal to $R\left[y^{t} ; \tau^{t}, \delta^{t}\right]$, and that $S$ is a free right $U$-module with basis $\left\{1, y, \ldots, y^{t-1}\right\}$. It thus follows directly from Theorem 
1.3 that all prime factors of $U$ have rank at most $b$. The theorem now follows from Lemma 1.4 .

Although the above result applies to the quantum coordinate rings $\mathscr{O}_{q}\left(M_{n}(k)\right)$ with $q$ a primitive $t$ th root of unity, there exist sharper bounds on Goldie ranks of prime factors than those obtainable from iterated use of Theorem 1.5-see Remark 3.5.

\section{ITERATED EXTENSIONS}

Sigurdsson's theorem (the special case of Theorem 1.3 in which $\tau=1$ ) extends to iterated differential operator rings of the form $S=R\left[y_{1} ; \delta_{1}\right]\left[y_{2} ; \delta_{2}\right] \cdots$ $\left[y_{n} ; \delta_{n}\right]$. Namely, if $R$ is a noetherian $\mathbb{Q}$-algebra and if there is a finite upper bound $b$ for the Goldie ranks of all prime factors of the polynomial ring $R[z]$, then $b$ is also an upper bound for the Goldie ranks of the prime factors of $S$ $[19,2.5]$. The proof relies on a result of Bell [2, Theorem A], saying that the Goldie ranks of the prime factors of the polynomial ring $R\left[z_{1}, \ldots, z_{n}\right]$ are bounded above by $b$. It also depends on the trivial fact that $R\left[z_{1}, \ldots, z_{n}\right]$ can be rewritten with the variables $z_{i}$ listed in any desired order. Of course, $R\left[z_{1}, \ldots, z_{n}\right]$ is just the associated graded ring of $S$ with respect to a natural filtration. However, reordering of variables is in general not possible for the associated graded ring of an iterated skew polynomial ring of the form $R\left[y_{1} ; \tau_{1}, \delta_{1}\right]\left[y_{2} ; \tau_{2}, \delta_{2}\right] \cdots\left[y_{n} ; \tau_{n}, \delta_{n}\right]$. Due to this obstacle, we develop an iterated version of Theorem 1.3 only for a special class of algebras.

We thank D. Passman for showing us how to obtain the following theorem from Roseblade's classification of prime ideals in group algebras of polycyclicby-finite groups [18]. We provide a direct proof, since the full machinery of [18] is not needed for the situation with which we deal.

Theorem 2.1. Let $k$ be a field, $\lambda=\left(\lambda_{i j}\right)$ an $n \times n$ matrix of nonzero elements of $k$ such that $\lambda_{i i}=1$ and $\lambda_{j i}=\lambda_{i j}^{-1}$ for $1 \leq i, j \leq n$, and $A(\lambda)$ the $k$-algebra generated by elements $x_{1}, \ldots, x_{n}$ subject only to the relations $x_{i} x_{j}=\lambda_{i j} x_{j} x_{i}$ for $1 \leq i, j \leq n$. Assume that the subgroup $\Lambda$ of $k^{\times}$generated by the $\lambda_{i j}$ is torsionfree. Then all of the prime ideals of $A(\lambda)$ are completely prime.

Proof. We proceed by induction on $n$, the case $n=1$ being trivial. We therefore assume that $n>1$ and that the theorem holds for all algebras of the given form on $n-1$ generators.

Let $P \in \operatorname{spec} A(\lambda)$. To begin, if $P$ contains one of the generators $x_{i}$, then $A(\lambda) / P$ is isomorphic to a prime factor of $A(\gamma)$, where $\gamma$ is the $(n-1) \times(n-1)$ matrix obtained from $\lambda$ by deleting the $i$ th row and column. In this case, the desired conclusion follows fom the induction hypothesis.

Now assume that $x_{i} \notin P$ for all $i$. Since the $x_{i}$ are normal elements, they generate a denominator set $X$ in $A(\lambda)$; the localization $A(\lambda) X^{-1}$ is just the $k$-algebra generated by $x_{1}, x_{1}^{-1}, \ldots, x_{n}, x_{n}^{-1}$ subject to the relations $x_{i} x_{j}=$ $\lambda_{i j} x_{j} x_{i}$. We denote this localization by $P(\lambda)$ as in [14]. The normality of the $x_{i}$ ensures that $X$ is disjoint from $P$, and so $P$ induces a prime ideal $Q$ in $P(\lambda)$. Therefore, it suffices to show that $Q$ is completely prime.

Let $G$ denote the subgroup of the group of units of $P(\lambda)$ generated by $x_{1}, \ldots, x_{n}$. The commutator subgroup of $G$ is $\Lambda$, and $G / \Lambda$ is free abelian of rank $n$. Let $H$ be the set of those $z \in G$ such that the coset $z+Q$ lies in the 
center of $P(\lambda) / Q$; then $H$ is a normal subgroup of $G$ containing $\Lambda$. Let $T$ be the $k$-subalgebra of $P(\lambda)$ generated by $H$. Since $T /(T \cap Q)$ embeds in the center of $P(\lambda) / Q$, it is a commutative domain. Let $K$ be the quotient field of $T /(T \cap Q)$, and observe that the localization $A=[P(\lambda) / Q] \mathscr{C}^{-1}$ with respect to the set $\mathscr{C}$ of nonzero elements of $T /(T \cap Q)$ is a $K$-algebra. To prove the theorem it suffices to demonstrate that $A$ is a domain.

We next claim that the group $G / H$ is torsionfree. First assume to the contrary that there exists an element $z \in G \backslash H$ such that $z^{m} \in H$ for some positive integer $m$. Since $z \notin H$, there is an index $j$ such that $z$ does not commute, modulo $Q$, with $x_{j}$. Now $z x_{j}=\lambda x_{j} z$ for some $\lambda \in \Lambda$, and so $\lambda \neq 1$; moreover, $\lambda^{m} \neq 1$ because $\Lambda$ is torsionfree. On the other hand, $z^{m} x_{j}=\lambda^{m} x_{j} z^{m} \equiv \lambda^{m} z^{m} x_{j}(\bmod Q)$ because $z^{m} \in H$. But then $Q$ contains the unit $\left(1-\lambda^{m}\right) z^{m} x_{j}$, which is impossible. Thus $G / H$ is torsionfree, as claimed.

In view of the preceding two paragraphs, $G / H$ is a free abelian group of rank at most $n$. Choose elements $y_{1}, \ldots, y_{m} \in G$ such that the cosets $y_{i} H$ form a basis for $G / H$. Observe that $A$ is generated as a $K$-algebra by the algebra cosets $\left(y_{1}+Q\right)^{ \pm 1}, \ldots,\left(y_{m}+Q\right)^{ \pm 1}$. Furthermore, for $1 \leq i, j \leq m$, there is a commutation relation $y_{i} y_{j} y_{i}^{-1} y_{j}^{-1}+Q=\mu_{i j}$ for some $\mu_{i j} \in K^{\times}$, where $\mu_{i i}=1$. Therefore, there exists a $K$-algebra epimorphism

$$
P(\boldsymbol{\mu})=K\left\{Y_{1}^{ \pm 1}, \ldots, Y_{m}^{ \pm 1}\right\} /\left\langle Y_{i} Y_{j}-\mu_{i j} Y_{j} Y_{i} \mid 1 \leq i, j \leq m\right\rangle \rightarrow A,
$$

where $\mu$ denotes the matrix $\left(\mu_{i j}\right)$ and $Y_{i} \mapsto y_{i}+Q$ for $1 \leq i \leq m$.

The final step is to apply $[14,1.3]$ to show that $P(\mu)$ is a simple ring. This requires showing that if $(r(1), \ldots, r(m))$ is any nonzero vector in $\mathbb{Z}^{m}$, then

$$
\mu_{1 j}^{r(1)} \mu_{2 j}^{r(2)} \cdots \mu_{m j}^{r(m)} \neq 1
$$

for some $j$. Now the monomial $y_{1}^{r(1)} y_{2}^{r(2)} \cdots y_{m}^{r(m)} \in G$ is not an element of $H$, since $G / H$ is a free abelian group on the cosets $y_{i} H$, and so $y_{1}^{r(1)} y_{2}^{r(2)} \cdots y_{m}^{r(m)}+$ $Q$ is not a central element of $P(\lambda) / Q$. It follows that $Y_{1}^{r(1)} Y_{2}^{r(2)} \cdots Y_{m}^{r(m)}$ cannot be central in $P(\mu)$. Consequently, there is an index $j$ such that $\mu_{1 j}^{r(1)} \mu_{2 j}^{r(2)} \ldots$ $\mu_{m j}^{r(m)} \neq 1$, as desired. We therefore conclude from [14, 1.3] that $P(\boldsymbol{\mu})$ is a simple ring and consequently that $A \cong P(\mu)$. However, $A$ is now seen to be an integral domain since $P(\boldsymbol{\mu})$ is an iterated skew Laurent extension of $K$. The theorem follows.

Remark 2.2. The hypothesis in Theorem 2.1 that $\Lambda$ be torsionfree is essential. For example, suppose that $k$ is a field whose characteristic is not 2 and that $q$ is a nonzero element of $k$ which is not a root of unity, and let

$$
\lambda=\left(\begin{array}{ccc}
1 & 1 & q \\
1 & 1 & -q \\
q^{-1} & -q^{-1} & 1
\end{array}\right)
$$

(Observe that $-1 \in \Lambda$.) The ideal $\left(x_{1}^{2}-x_{2}^{2}\right) A(\lambda)$ is a prime ideal of $A(\lambda)$ which is not completely prime. 
Theorem 2.3. Let $S=k\left[y_{1}\right]\left[y_{2} ; \tau_{2}, \delta_{2}\right] \cdots\left[y_{n} ; \tau_{n}, \delta_{n}\right]$ be an iterated skew polynomial ring over a field $k$. For $1 \leq i \leq n$ set $S_{i}=k\left[y_{1}\right]\left[y_{2} ; \tau_{2}, \delta_{2}\right] \cdots$ $\left[y_{i} ; \tau_{i}, \delta_{i}\right]$, and for $2 \leq i \leq n$ assume that the following conditions hold:

(a) $\tau_{i}$ is a k-algebra automorphism of $S_{i-1}$, and $\delta_{i}$ is a k-linear $\tau_{i^{-}}$ derivation of $S_{i-1}$.

(b) For each $j=1, \ldots, i-1$ there exists $\lambda_{i j} \in k^{\times}$such that $\tau_{i}\left(y_{j}\right)=\lambda_{i j} y_{j}$.

(c) There exists $q_{i} \in k^{\times}$such that $\delta_{i} \tau_{i}\left(y_{j}\right)=q_{i} \tau_{i} \delta_{i}\left(y_{j}\right)$ for $j=1, \ldots$, $i-1$.

(d) Either $q_{i}$ is not a root of unity, or $q_{i}=1$ and the characteristic of $k$ is zero.

Further assume that the subgroup $\Lambda$ of $k^{\times}$generated by the $\lambda_{i j}$ is torsionfree. Then all prime ideals of $S$ are completely prime.

Proof. For $1 \leq m \leq n$ let $\mathbb{T}_{m}$ denote the statement that the theorem holds in the case that $\delta_{i}=0$ for all $i>m$. We shall prove statements $\mathbb{T}_{1}, \ldots, \mathbb{T}_{n}$ by induction, statement $\mathbb{T}_{n}$ being the full theorem. To begin, observe that statement $\mathbb{T}_{1}$ follows from Theorem 2.1.

Now fix $2 \leq m \leq n$, and assume that statement $\mathbb{T}_{m-1}$ holds. To prove $\mathbb{T}_{m}$, we may assume that $\delta_{m+1}, \ldots, \delta_{n}$ all vanish. It follows that $y_{i} y_{j}=\lambda_{i j} y_{j} y_{i}$ for $m+1 \leq i \leq n$ and $1 \leq j \leq i-1$. Consequently, the $k$-subalgebra $R$ of $S$ generated by $S_{m-1} \cup\left\{y_{m+1}, \ldots, y_{n}\right\}$ is an iterated skew polynomial ring of the form

$$
R=S_{m-1}\left[y_{m+1} ; \tau_{m+1}^{\prime}\right] \cdots\left[y_{n} ; \tau_{n}^{\prime}\right],
$$

where $\tau_{i}^{\prime}$ denotes the restriction of $\tau_{i}$ to the algebra generated by $S_{m-1} \cup$ $\left\{y_{m+1}, \ldots, y_{i-1}\right\}$. Now $y_{m} y_{j}=\lambda_{m j} y_{j} y_{m}+\delta_{m}\left(y_{j}\right)$ for $1 \leq j \leq m-1$ and $y_{m} y_{i}=\lambda_{i m}^{-1} y_{i} y_{m}$ for $m+1 \leq i \leq n$. It follows that $S$ can be expressed in the form $S=R\left[y_{m} ; \tau, \delta\right]$, where $\tau$ is a $k$-algebra automorphism of $R$ such that

$$
\tau\left(y_{j}\right)= \begin{cases}\lambda_{m j} y_{j} & \text { for } 1 \leq j \leq m-1, \\ \lambda_{j m}^{-1} y_{j} & \text { for } m+1 \leq j \leq n\end{cases}
$$

and $\delta$ is a $k$-linear $\tau$-derivation of $R$ such that

$$
\delta\left(y_{j}\right)= \begin{cases}\delta_{m}\left(y_{j}\right) & \text { for } 1 \leq j \leq m-1, \\ 0 & \text { for } m+1 \leq j \leq n .\end{cases}
$$

Next, consider the algebra

$$
R[z ; \tau]=S_{m-1}\left[y_{m+1} ; \tau_{m+1}^{\prime}\right] \cdots\left[y_{n} ; \tau_{n}^{\prime}\right][z ; \tau] .
$$

It follows from statement $\mathbb{T}_{m-1}$ that all prime ideals in $R[z ; \tau]$ are completely prime.

Observe for each $j=1, \ldots, m-1$ that $\tau_{m}^{-1} \delta_{m} \tau_{m}\left(y_{j}\right)=q_{m} \delta_{m}\left(y_{j}\right)$. Since the restrictions of $\tau$ and $\delta$ to $S_{m-1}$ agree with $\tau_{m}$ and $\delta_{m}$, we thus have $\tau^{-1} \delta \tau\left(y_{j}\right)=q_{m} \delta\left(y_{j}\right)$ for $j=1, \ldots, m-1$. On the other hand, $\tau^{-1} \delta \tau\left(y_{j}\right)=$ $0=q_{m} \delta\left(y_{j}\right)$ for $j=m+1, \ldots, n$, and hence the $\tau$-derivations $\tau^{-1} \delta \tau$ and $q_{m} \delta$ agree on a set of $k$-algebra generators for $R$. It follows that $\tau^{-1} \delta \tau=q_{m} \delta$, that is, $\delta \tau=q_{m} \tau \delta$. Furthermore, assumption (d) implies that $q_{m}^{r}+q_{m}^{r-1}+\cdots$ +1 is invertible in $k$ for all positive integers $r$. Since all prime ideals in $R[z ; \tau]$ are completely prime, we therefore conclude from Theorem 1.3 that all prime ideals in $S$ are completely prime. This completes the induction step. 


\section{QUANTUM COORDINATE RINGS}

Throughout this section, $k$ denotes a field, $q$ a nonzero element of $k$, and $n$ a positive integer. The following presentations for the quantum algebras $\mathscr{O}_{q}\left(M_{n}(k)\right)$ are taken from [20]. To obtain the presentations in [17], for instance, replace $q^{2}$ by $q^{-1}$.

3.1. The coordinate ring of quantum $n \times n$ matrices over $k$, denoted $\mathscr{O}_{q}\left(M_{n}(k)\right)$, is the $k$-algebra generated by elements $x_{i j}$ for $i, j=1, \ldots, n$ subject to the following relations:

$$
\begin{aligned}
x_{i j} x_{\ell j} & =q^{2} x_{\ell j} x_{i j} & & \text { when } i<\ell ; \\
x_{i j} x_{i m} & =q^{2} x_{i m} x_{i j} & & \text { when } j<m ; \\
x_{i m} x_{\ell j} & =x_{\ell j} x_{i m} & & \text { when } i<\ell \text { and } j<m ; \\
x_{i j} x_{\ell m}-x_{\ell m} x_{i j} & =\left(q^{2}-q^{-2}\right) x_{i m} x_{\ell j} & & \text { when } i<\ell \text { and } j<m .
\end{aligned}
$$

If the $x_{i j}$ are adjoined in lexicographic order, direct calculations show that $\mathscr{O}_{q}\left(M_{n}(k)\right)$ is an iterated skew polynomial ring

$$
k\left[x_{11}\right]\left[x_{12} ; \tau_{12}\right] \cdots\left[x_{i j} ; \tau_{i j}, \delta_{i j}\right] \cdots\left[x_{n n} ; \tau_{n n}, \delta_{n n}\right],
$$

where each $\tau_{\ell m}$ and each $\delta_{\ell m}$ is $k$-linear and

$$
\begin{aligned}
& \tau_{\ell m}\left(x_{i j}\right)= \begin{cases}q^{-2} x_{i j} & \text { when } i=\ell \text { and } j<m, \\
q^{-2} x_{i j} & \text { when } i<\ell \text { and } j=m, \\
x_{i j} & \text { when } i<\ell \text { and } j \neq m,\end{cases} \\
& \delta_{\ell m}\left(x_{i j}\right)= \begin{cases}0 & \text { when } i=\ell \text { and } j<m, \\
0 & \text { when } i<\ell \text { and } j \geq m, \\
\left(q^{-2}-q^{2}\right) x_{i m} x_{\ell j} & \text { when } i<\ell \text { and } j<m .\end{cases}
\end{aligned}
$$

Observe that $\delta_{\ell m} \tau_{\ell m}\left(x_{i j}\right)=q^{4} \tau_{\ell m} \delta_{\ell m}\left(x_{i j}\right)$ when $(i, j)<(\ell, m)$ in the lexicographic order. (In particular, it follows that $\delta_{\ell m} \tau_{\ell m}=q^{4} \tau_{\ell m} \delta_{\ell m}$.) Finally, note that if $q$ is not a root of unity, then the subgroup of $k^{\times}$generated by $q^{-2}$ is torsionfree.

We now obtain our main result directly from Theorem 2.3.

Theorem 3.2. If $q$ is not a root of unity, then all prime ideals of $\mathscr{O}_{q}\left(M_{n}(k)\right)$ are completely prime.

Corollary 3.3. If $q$ is not a root of unity, then all prime ideals of $\mathscr{O}_{q}\left(\mathrm{GL}_{n}(k)\right)$ and $\mathscr{O}_{q}\left(\mathrm{SL}_{n}(k)\right)$ are completely prime.

Proof. The algebra $\mathscr{O}_{q}\left(\mathrm{GL}_{n}(k)\right)$ is a localization of $\mathscr{O}_{q}\left(M_{n}(k)\right)$, while $\mathscr{O}_{q}\left(\mathrm{SL}_{n}(k)\right)$ is a factor of $\mathscr{O}_{q}\left(M_{n}(k)\right)$. (See [20] for details.)

Remarks 3.4. Assume that $q \in \mathbb{C}^{\times}$is not a root of unity.

(i) For $\mathscr{O}_{q}\left(\mathrm{SL}_{3}(\mathbb{C})\right.$ ), complete primeness of the prime ideals is proved in [8].

(ii) Complete primeness of the co-finite-dimensional maximal ideals of $\mathscr{O}_{q}\left(M_{n}(\mathbb{C})\right.$ ) is proved in [9]. (Observe that this statement is equivalent to the assertion that all finite dimensional irreducible representations are one dimensional.) 
(iii) An immediate corollary of Theorem 2.1 is that all prime ideals in $\mathscr{O}_{q}\left(\mathbb{C}^{n}\right)$ are completely prime. This fact is proved in [16].

Remark 3.5. Suppose that $q \in k^{\times}$is a primitive $t$ th root of unity, with $t$ odd. In this case, the $t$ th powers $x_{i j}^{t}$ are central in $\mathscr{O}_{q}\left(M_{n}(k)\right)$ ([17, 7.2.1]; cf. [6, 14.11]), and so $\mathscr{O}_{q}\left(M_{n}(k)\right)$ is a free module of rank $t^{n^{2}}$ over a central noetherian subring. It follows from standard arguments that the prime factors of $\mathscr{O}_{q}\left(M_{n}(k)\right)$ all have Goldie rank at most $t^{n^{2} / 2}$ (see, e.g., $\left.[6,14.10]\right)$. For the case when $n=2$, the minimal bound is $t[6,14.14]$.

Remark 3.6. We can also apply our methods to the multiparametrizations studied in $[1,13,21]$. In particular, if the subgroup of $k^{\times}$generated by the parameters is torsionfree, then it follows from Theorem 2.3 and additional calculations that every prime ideal of the multiparameter quantum coordinate ring of $M_{n}(k)$ is completely prime. For details, see the expository paper [5], which also presents a direct route through that portion of [6] used in proving Theorem 1.3. A proof that the multiparameter quantum coordinate ring of $\mathrm{SL}_{n}(k)$ is a domain, without the preceding hypothesis on the parameters, is presented in [12].

\section{ACKNOWLEDGMENT}

We thank S. P. Smith for calling our attention to the problem of determining the structure of prime factor algebras of quantum coordinate rings.

\section{REFERENCES}

1. M. Artin, W. Schelter, and J. Tate, Quantum deformations of $G L_{n}$, Comm. Pure Appl. Math. 44 (1991), 879-895.

2. A. D. Bell, Goldie dimension of prime factors of polynomial and skew polynomial rings, J. London Math. Soc. (2) 29 (1984), 418-424.

3. A. W. Goldie and G. O. Michler, Ore extensions and polycyclic group rings, J. London Math. Soc. (2) 9 (1974), 337-345.

4. K. R. Goodearl, Prime ideals in skew polynomial rings and quantized Weyl algebras, J. Algebra 150 (1992), 324-377.

5. __ Uniform ranks of prime factors of skew polynomial rings, Ring Theory, Proc. Biennial Ohio State-Denison Conf., 1992 (S. K. Jain and S. T. Rizvi, eds.), World Scientific, Singapore, 1993, pp. 182-199.

6. K. R. Goodearl and E. S. Letzter, Prime ideals in skew and q-skew polynomial rings, Mem. Amer. Math. Soc., Amer. Math. Soc., Providence, RI (to appear).

7. K. R. Goodearl and R. B. Warfield, Jr., An introduction to noncommutative Noetherian rings, London Math. Soc. Student Texts, vol. 16, Cambridge Univ. Press, Cambridge, 1989.

8. T. J. Hodges and T. Levasseur, Primitive ideals of $\mathrm{C}_{q}[S L(3)]$, Comm. Math. Phys. (to appear).

9. S.-Q. Oh, Finite dimensional simple modules over the coordinate ring of quantum matrices, Bull. London Math. Soc. 25 (1993), 427-430.

10. A. V. Jategaonkar, Localization in Noetherian Rings, London Math. Soc. Lecture Note Ser., vol. 98, Cambridge Univ. Press, Cambridge, 1986.

11. E. S. Letzter, Finite correspondence of spectra in noetherian ring extensions, Proc. Amer. Math. Soc. 116 (1992), 645-652.

12. T. Levasseur and J. T. Stafford, The quantum coordinate ring of the special linear group, J. Pure Appl. Algebra 86 (1993), 181-186. 
13. Yu. I. Manin, Multiparametric quantum deformation of the general linear supergroup, Comm. Math. Phys. 123 (1989), 163-175.

14. J. C. McConnell and J. J. Pettit, Crossed products and multiplicative analogues of Weyl algebras, J. London Math. Soc. (2) 38 (1988), 47-55.

15. J. C. McConnell and J. C. Robson, Noncommutative Noetherian Rings, Wiley-Interscience, New York, 1987.

16. S.-Q. Oh, Doctoral dissertation, Univ. of Cincinnati, 1992.

17. B. Parshall and J.-P. Wang, Quantum linear groups, Mem. Amer. Math. Soc., vol. 89, Amer. Math. Soc., Providence, RI, 1991.

18. J. E. Roseblade, Prime ideals in group rings of polycyclic groups, Proc. London Math. Soc. (3) 36 (1978), 385-447.

19. G. Sigurdsson, Differential operator rings whose prime factors have bounded Goldie dimension, Arch. Math. (Basel) 42 (1984), 348-353.

20. S. P. Smith, Quantum groups: An introduction and survey for ring theorists, Noncommutative Rings (S. Montgomery and L. Small, eds.), Math. Sci. Res. Inst. Publ., vol. 24, Springer-Verlag, New York, 1992, pp. 131-178.

21. A. Sudbury, Consistent multiparameter quantisation of $G L(n)$, J. Phys. A 23 (1990), L697-L704.

Department of Mathematics, University of California, Santa Barbara, California 93106

E-mail address: goodearl@math.ucsb.edu

Department of Mathematics, University of Utah, Salt Lake City, Utah 84112

Current address: Department of Mathematics, Texas A\&M University, College Station, Texas 77843

E-mail address: letzter@math.tamu.edu 